AdAM, K. M. G. (1959). J. gen. Microbiol. 21, 519-529

\title{
The Growth of Acanthamoeba sp. in a Chemically Defined Medium
}

\author{
BY KATHERINE M. G. ADAM \\ Department of Zoology, University of Edinburgh
}

\begin{abstract}
SUMMARY: A study of the nutritional requirements of Acanthamoeba sp. Neff. has led to the development of a chemically defined medium which contains 18 amino acids, vitamin $B_{12}$ and thiamin, acetate, citric acid, salts and trace metals. The need for vitamin $B_{12}$ and thiamin only is an indication that Acanthamoeba may be closely related to the phytoflagellates. Growth of the amoebae in the defined medium is similar to the growth in peptone, but slower. Glucose in high concentration replaced acetate in the defined medium.
\end{abstract}

Many protozoa can now be grown axenically in chemically defined media. The species whose nutritional requirements are known are all flagellates with the exception of Tetrahymena pyriformis, a ciliate. Although several members of the Amoebida can be grown axenically, none so far appears to have been grown in a medium of known chemical composition.

\section{METHODS}

Organism. The amoeba used for the present investigation was isolated by Neff (1957) from soil at Pacific Grove, California. Axenic cultures of this amoeba were obtained from Dr S. H. Hutner (Haskins Laboratories, New York), where this work on its nutrition was started.

Cultivation. According to Neff (1957) this amoeba can be grown in a variety of liquid media, the most satisfactory being one which contains Proteose peptone, glucose and inorganic salts. Dr M. M. Swann (personal communication) observed that it also grew well in Mycological peptone (Oxoid) and that $4 \%(\mathrm{w} / \mathrm{v})$ was about the optimum concentration. Acanthamoeba sp. has been maintained in this laboratory since 1955 in $4 \%(w / v)$ Mycological peptone. Transfers are made every 2 weeks, but the interval may be safely prolonged to 4 weeks.

Glassware. All glassware was cleaned in concentrated sulphuric acid containing potassium dichromate, rinsed twelve times in hot tap water and then twice in glass-distilled water.

Chemicals. The amino acids, vitamins (except $\mathrm{B}_{12}$ ), purines and pyrimidines were obtained from Roche Products Ltd.; the remaining chemicals (AnalaR quality) from British Drug Houses Ltd. Cyanocobalamin (vitamin $B_{12}$ ) was kindly supplied by Glaxo Laboratories Ltd. as their preparation 'Cytamen'. Stock solutions of chemicals were stored at $5^{\circ}$ with volatile preservative added (Hutner, Provasoli \& Filfus, 1953). The amino acids were prepared as a dry mixture (Hutner, Bach \& Ross, 1956); the vitamins 
were similarly prepared with mannitol as triturate. Solutions of chemicals and media were prepared with twice glass-distilled water which had been stored with volatile preservative.

Media. Since Acanthamoeba sp. will grow in an aqueous solution of peptone which has been sterilized by autoclaving, it was assumed to require only watersoluble and heat-stable nutrients for growth. The composition of a basal defined medium (B.D.M.) containing only such nutrients is shown in Table 1. The amino acids and vitamins were added; the remaining chemicals were measured from stock solutions. Water was added to bring the basal medium to twice the desired concentration. Further nutrients could then be added in an equal volume of water to samples of the twice-concentrated medium, thereby adjusting the concentration of the constituents to that shown in Table 1.

Table 1. Composition of basal defined medium

\begin{tabular}{|c|c|c|c|}
\hline & $\begin{array}{c}\text { Concn } \\
(\mathrm{mg} \cdot / 100 \mathrm{ml} .)\end{array}$ & & $\begin{array}{c}\text { Concn. } \\
(\mu \mathrm{g} . / 100 \mathrm{ml} .)\end{array}$ \\
\hline Block $A$ : amino acids & & Block V: vitamins & \\
\hline L-Arginine $\mathbf{H C l}$ & $45 \cdot 0$ & Thiamin $\mathbf{H C l}$ & $500 \cdot 0$ \\
\hline L-Histidine, base & $\mathbf{3 2 \cdot 5}$ & Nicotinamide & $100 \cdot 0$ \\
\hline DL-Isoleucine & $25 \cdot 0$ & Ca-d-pantothenate & $100 \cdot 0$ \\
\hline L-Leucine & $25 \cdot 0$ & Pyridoxamine $2 \mathrm{HCl}$ & $100 \cdot 0$ \\
\hline L-Lysine $2 \mathrm{HCl}$ & $20 \cdot 0$ & Riboflavin & 50.0 \\
\hline DL-Methionine & $9 \cdot 0$ & Folic acid & $5 \cdot 0$ \\
\hline DL-Phenylalanine & $\mathbf{3 2 \cdot 0}$ & Biotin & 0.5 \\
\hline DL-Threonine & $20 \cdot 0$ & $\mathrm{~B}_{18}$ & $0 \cdot 1$ \\
\hline DL-Tryptophan & $\mathbf{2 5} \cdot 0$ & Pyridoxine & $100 \cdot 0$ \\
\hline DL-Valine & $17 \cdot 5$ & Choline chloride & $500 \cdot 0$ \\
\hline DL-Alanine & $\mathbf{3 5} \cdot 0$ & $p$-Aminobenzoic acid & $10 \cdot 0$ \\
\hline DL-Aspartic acid & $25 \cdot 0$ & Inositol & $1000 \cdot 0$ \\
\hline L-Cystine & $2 \cdot 5$ & Mannitol, as triturate & $2400 \cdot 0$ \\
\hline L-Glutamic acid & $50 \cdot 0$ & & \\
\hline Glycine & $25 \cdot 0$ & & (mg./100 ml.) \\
\hline L-Proline & $2 \cdot 0$ & Block $S$ : salts & \\
\hline DL-Serine & $\mathbf{3 5} \cdot \mathbf{0}$ & $\begin{array}{l}\mathrm{MgSO}_{4} .7 \mathrm{H}_{2} \mathrm{O} \\
\mathrm{Mg}\end{array}$ & $20 \cdot 0$ \\
\hline L-Tyrosine & $\mathbf{2 \cdot 0}$ & $\mathrm{CaCl}_{8}$ & $1 \cdot 0$ \\
\hline \multicolumn{2}{|c|}{ Block $P$ : purines and pyrimidines } & $\mathrm{KH}_{2} \mathrm{PO}_{4}$ & $20 \cdot 0$ \\
\hline Guanylic acid & $1 \cdot 0$ & $\mathrm{Na}_{2} \mathrm{HPO}_{4}$ & $20 \cdot 0$ \\
\hline Adenylic acid & $\mathbf{0 \cdot 5}$ & Trace metal solution* & $1 \mathrm{ml} / 100 \mathrm{ml}$. \\
\hline Cytidylic acid & 0.5 & Trace metal solution* & $1 \mathrm{ml} . / 100 \mathrm{ml}$. \\
\hline Uracil & $\mathbf{0} \cdot \mathbf{1}$ & & \\
\hline
\end{tabular}

* $1 \mathrm{ml} . / 100 \mathrm{ml}$. medium gives metal ion concentration: $\mu \mathrm{g}$, Fe 100, $\mathrm{Zn} \mathrm{22.6,} \mathrm{Mn} \mathrm{65,}$ Mo 20, $\mathrm{Co} 0 \cdot 63, \mathrm{Cu} 0 \cdot 13$, EDTA $\mathrm{Na}_{2}$ 1000. Salts used were all sulphates, Mo as ammonium molybdate. pH of medium adjusted to $c .6 \cdot 5$.

An aqueous extract of yeast autolysate (Y.A., Difco), which was used as supplement for the B.D.M., was prepared by suspending $10 \mathrm{~g}$. powder in $100 \mathrm{ml}$. water and boiling for $15 \mathrm{~min}$; water lost by evaporation was replaced. The cooled suspension was then filtered through a no. 1 Whatman filter paper. Evaporation of a sample of the filtrate to dryness and constant weight showed that it contained $c .60 \mathrm{mg}$. solute $/ \mathrm{ml}$.

The pH value of media was adjusted before autoclaving to 6.5 (B.D.H. 
indicator paper, $5 \cdot 5-7 \cdot 0$ ) by the addition of $\mathrm{N}-\mathrm{NaOH}$. In experiments to test the effect of $\mathrm{pH}$ on growth, the $\mathrm{pH}$ was determined electrometrically to within $\pm 0 \cdot 1$ unit.

Media were autoclaved for $30 \mathrm{~min}$. at $15 \mathrm{lb}$./sq.in. Glucose was autoclaved separately.

Growth tests. In the early part of this work, the amoebae were cultured in $10 \mathrm{ml}$. medium placed in $20 \times 125 \mathrm{~mm}$. screw-capped test tubes. The tubes were incubated at $23^{\circ}$ and left undisturbed for 3 weeks, which was taken as the standard growth period. In most experiments the tubes were inoculated in duplicate and from the same culture. The inoculum size was adjusted to give an initial concentration of 1-5 organisms $/ \mathrm{mm}^{3}$. Growth was considered to have occurred when the concentration of cells at end of the growth period was about 100 cells $/ \mathrm{mm}^{3}$. In later experiments, when it was desired to follow the rate of growth in more detail, the organisms were grown in $50 \mathrm{ml}$. conical flasks which contained $10 \mathrm{ml}$. medium and which were plugged with cotton wool. Serial samples were taken from replicated cultures at intervals of 2-3 days over a period of 2-4 weeks.

Checks for sterility were made from time to time by inoculating samples of cultures into a variety of liquid and solid media which were incubated aerobically and anaerobically.

Counting. Growth was estimated by counting live amoebae in a FuchsRosenthal haemocytometer. The tube or flask was shaken vigorously for a definite number of times to dislodge the cells and a sample was immediately transferred with a clean, sterile Pasteur pipette to the counting chamber. Unless the suspension contained more than 1000 cells per $3 \mathrm{~mm} .^{3}$, all the organisms in $3 \mathrm{~mm} .{ }^{3}$ were counted and the results expressed as the number of amoebae $/ \mathrm{mm} .^{3}$ of medium. The counting error was small and could be neglected in counts of less than 1000 organisms. With higher counts the sample was diluted whenever this was possible. This method was tested by following the distribution of cells in the counting chamber and then applying a Poisson calculation to the data (Fisher, 1941). Nineteen samples taken from six cultures containing different concentrations of organisms $\left(30-260\right.$ cells $\left./ \mathrm{mm}^{3}{ }^{3}\right)$ were examined. The observed distribution was found to differ significantly $(P<0.05)$ from the expected in five out of the nineteen samples. Four of the significant differences occurred in samples containing less than 100 organisms $/ \mathrm{mm}^{3}$; it was concluded that the method was adequate for concentrations in the range 100-260 organisms $/ \mathrm{mm}^{3}$. When replicated counts were made on single cultures in this range, differences larger than would be expected from the error of random sampling occurred with a frequency of about 1 in 10 counts. Closer agreement with the Poisson distribution might have been achieved by performing the counts on killed organisms (Neff, Neff \& Taylor, 1958), since live organisms tend to form clumps or adhere to glass. In experiments on growth rate, the cultures were replicated so that the mean with its standard error could be calculated for each point on the curve. 


\section{RESULTS}

Development of a defined medium. The basal defined medium (B.D.M., 'Table 1) did not by itself support growth. However, when it was enriched with an aqueous extract of yeast autolysate (Y.A., $8 \%, \mathrm{v} / \mathrm{v}$ ), the yield was 100-200 organisms $/ \mathrm{mm}^{3}$ at the end of 3 weeks. Since the B.S.M. did not contain a carbohydrate source of energy, acetate and glucose were tested as possible replacements for the Y.A. When acetate $(0.25 \%, \mathrm{w} / \mathrm{v}$, as the sodium salt) was added to the medium it was possible to decrease the X.A. from 8 to $4 \%(v / v)$ and still obtain the same amount of growth. Glucose $(0.25 \%, w / v)$ had no such sparing action. Acetate was therefore added to the B.D.M. in all subsequent experiments.

In a further series of experiments the concentration of the medium as a whole, or of the separate blocks, was varied in combination with smaller concentrations of Y.A. It was found that by raising the concentration of the salts (block S) to four times that shown in Table 1, the organisms grew in presence of only $2 \%(\mathrm{v} / \mathrm{v})$ Y.A. Block $\mathrm{S}$ of the B.D.M. was subsequently used at this increased concentration. The results of other tests were negative and it seemed unlikely that the Y.A. could be decreased further, still less eliminated, by varying the concentrations of nutrients in the B.D.M. At the suggestion of Dr M. R. Droop (the Marine Station, Millport), citric acid (1000 $\mu \mathrm{g}$.) was substituted for ethylenediamine tetra-acetic acid, di-sodium salt (EDTA) in the trace metal solution; at the same time citric acid was added to the medium. Under these conditions the amoebae grew without the addition of Y.A. Table 2 shows that slow growth was maintained through three subcultures in the modified B.D.M. containing $(0 \cdot 1 \%, \mathrm{w} / \mathrm{v})$ citric acid.

Table 2. The effect of adding citric acid to the basal defined medium*

\begin{tabular}{|c|c|c|c|c|c|}
\hline & \multicolumn{5}{|c|}{ Amoebae $/ \mathrm{mm}^{3}{ }^{3}$} \\
\hline & \multirow{2}{*}{$\begin{array}{c}\text { 1st transfer } \\
\text { (weeks) } \\
\mathbf{3}\end{array}$} & \multicolumn{2}{|c|}{$\begin{array}{l}\text { 2nd transfer } \\
\text { (weeks ) }\end{array}$} & \multicolumn{2}{|c|}{$\begin{array}{l}\text { 3rd transfer } \\
\text { (weeks) }\end{array}$} \\
\hline & & $\mathbf{3}$ & 4 & $\mathbf{3}$ & 4 \\
\hline B.D.M. & $\mathbf{3 4}$ & - & - & - & - \\
\hline $\begin{array}{l}\text { B.D.M. + citric acid } \\
\text { 0.01\%(w/v) }\end{array}$ & 78 & 20 & - & 11 & - \\
\hline $\begin{array}{l}\text { B.D.M. + citric acid } \\
0.1 \%(w / v)\end{array}$ & 92 & 56 & 80 & 36 & 60 \\
\hline
\end{tabular}

* Basal defined medium as in Table 1 with block $S \times 4$, citric acid replacing EDTA in trace metal solution $+0.25 \%(w / v)$ sodium acetate.

\section{Simplification of the defined medium}

(a) Purines and pyrimidines. The omission of block $\mathbf{P}$ did not impair growth and since the amoebae have been carried through at least twelve subcultures in media without purines and pyrimidines it is concluded that they can synthesize these substances.

(b) Vitamins. Initial experiments on vitamin requirements seemed to indicate 
that only vitamin $B_{12}$ was essential for growth. However, in media prepared with $B_{12}$ as the only vitamin, the yield of organisms dropped in the third subculture and growth was absent in the fourth. The results of further experiments, which are presented in Table 3, showed that thiamin was also essential for growth. It was subsequently confirmed that if either $\mathbf{B}_{12}(0 \cdot 1 \mu \mathrm{g} . \%, \mathrm{w} / \mathrm{v})$ or thiamin $(0.1 \mathrm{mg} . \%)$ was omitted from the medium, growth ceased in the second transfer.

\section{Table 3. Analysis of vitamin requirements}

Tubes were inoculated from a culture which had been subcultured three times in a medium containing $B_{12}$ as the only vitamin.

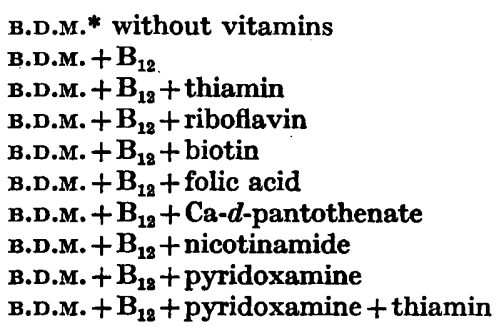

$\begin{array}{cc}\text { Amoebae } / \mathbf{m m}^{3}{ }^{3} & \text { after } 3 \text { weeks } \\ 2 & \text { Tube B } \\ 2 & 0 \\ 131 & 3 \\ 2 & 118 \\ 6 & 2 \\ 1 & 2 \\ 2 & 1 \\ 1 & 2 \\ 2 & 3 \\ 162 & 1 \\ & 160\end{array}$

* B.D.M. as in Table 1 with blocks $P$ and $V$ omitted, block $S \times 4$, citric acid replacing EDTA in trace metal solution, + sodium acetate $0.25 \%$, + citricacid $0 \cdot 1 \%(w / v)$. The vitamins were added at concentrations as in Table 1.

(c) Amino acids. Ammonium nitrate $(0 \cdot 25 \%, w / v)$ was substituted for block $\mathbf{A}$ of the modified and simplified B.D.M. No growth occurred. The ten amino acids considered essential for the growing rat (block $\mathbf{A}$ : arginine to valine inclusive) were next tried, but these also failed to support growth. Each of the remaining 8 amino acids was added to a medium containing the ten essential acids. Growth occurred only in tubes containing glycine, but it was less than in the control tubes in which all 18 amino acids were present. The optimum concentration of block $\mathbf{A}$ was found to be three times that shown in Table 1. A detailed analysis of the nitrogen requirements is now in progress:

Hydrogen-ion concentration. The effect of $\mathrm{pH}$ value on growth in modified and simplified B.D.M. was tested in the range 5.5-7.5. Growth was equivalent in the tubes in which the medium had been adjusted to $\mathrm{pH} 6.0,6.5$ and $7 \cdot 0$, but was decreased at pH $5 \cdot 5$ and $7 \cdot 5$.

Chelating agents. When citric acid (other than that in the trace metal solution) was omitted from the simplified defined medium, a precipitate formed on autoclaving. This precipitate could be avoided by decreasing the concentration of the $\mathrm{Ca}$ and $\mathrm{Mg}$ salts from 4 and $80 \mathrm{mg}$. $\%(\mathrm{w} / \mathrm{v})$ to 0.4 and $8.0 \mathrm{mg}$. $\%$, respectively; growth was not affected. Nevertheless, when these concentrations were slightly exceeded some precipitation was likely to occur on autoclaving. When the medium contained $\mathrm{Ca}$ and $\mathrm{Mg}$ salts at 4 and $80 \mathrm{mg} . \%$, the minimal concentration of citric acid required to prevent precipitation was $0.05 \%$ $(w / v)$; growth was not affected. Similarly, the citric acid could be increased 
to $\mathbf{0 . 2} \%$ without depressing growth. However, when the concentration of citric acid was raised to $0.5 \%$ the yield of organisms was halved as compared with control tubes which contained only $0.1 \%$ citric acid.

It was not found possible to substitute EDTA for citric acid in the present medium and obtain optimum growth. The least concentration of EDTA required to prevent precipitation on autoclaving was $15 \mathrm{mg} . \%(\mathrm{w} / \mathrm{v})$, but at this concentration growth was decreased. When the concentration of EDTA was increased to $30 \mathrm{mg}$. $\%$, growth was absent. At this concentration there was enough EDTA to combine with all the $\mathrm{Ca}^{++}$but not all the $\mathrm{Mg}^{++}$in the medium; the addition of graded amounts of $\mathrm{CaCl}_{2}$ to a medium containing $30 \mathrm{mg} . \%$ EDTA restored growth but the yield was still diminished.

Growth curves. It was now possible to compare the growth of Acanthamoeba sp. in peptone with that in a chemically defined medium. The results of growing the organisms are shown in Fig. 1. Serial counts were made on five tubes. Since the counts at zero time were likely to vary from 1 to 5 organisms $/ \mathrm{mm} .^{3}$, the

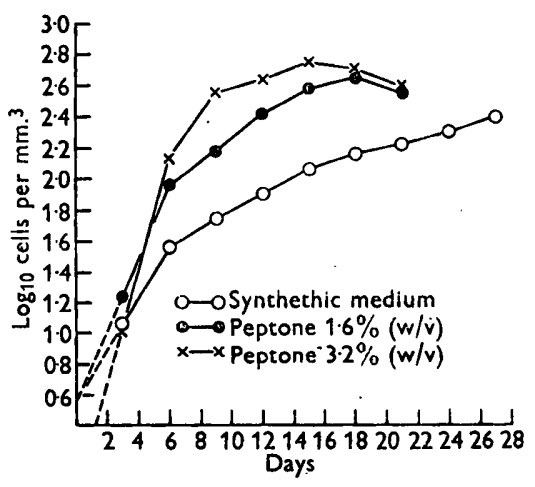

Fig. 1

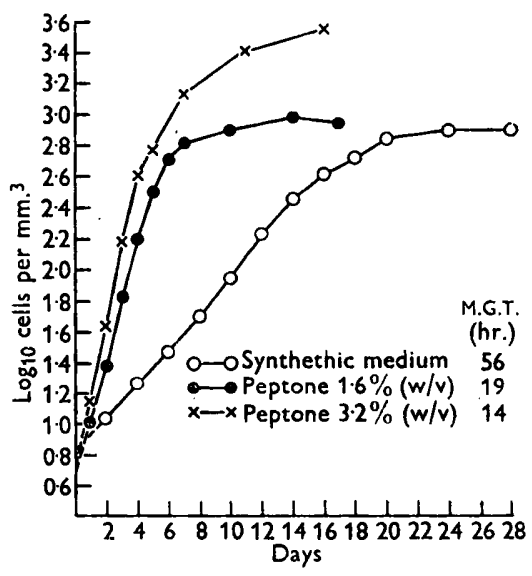

Fig. 2

Fig. 1. Growth of Acanthamoeba sp. in test tubes at $\mathbf{2 3}^{\circ}$ in two concentrations of mycological peptone and in defined medium. Amino acids as in Table $1, \times 3$, vitamin $\mathrm{B}_{12} 0.1 \mu \mathrm{g} . \%$, thiamin $0.1 \mathrm{mg} . \%$, salts as in Table $1, \times 4$, trace metals as in Table 1 with citric acid replacing EDTA $5 \mathrm{ml}$. \%, citric acid $0.1 \mathrm{~g} . \%$, sodium acetate $0.1 \mathrm{~g} . \%$.

Fig. 2. Growth of Acanthamoeba sp. in flasks at $23^{\circ}$ in two concentrations of Mycological peptone and in defined medium (composition, see Fig. 1). M.G.T. = mean generation time for logarithmic phase of growth.

initial part of each curve is of uncertain value and is shown with a broken line. Figure 2 shows the curves obtained when the amoebae were grown in $50 \mathrm{ml}$. conical flasks. Each point on the part of the curve representing logarithmic growth is the mean value obtained from eight flasks for each medium; thereafter only five flasks were used. Organisms used to inoculate tubes or flasks were taken from cultures already established in similar media. It was apparent that growth in the defined medium was comparable with that in Mycological peptone but that the rate of growth was slower. When enough time was allowed, 
the population size in the defined medium approached that in $1.6 \%(\mathrm{w} / \mathrm{v})$ peptone. Growth during the log. phase could be studied satisfactorily only when the organisms were grown in flasks. The numerical results for the defined medium are shown in Table 4. Exponential growth stopped at an approximately equivalent cell concentration $\left(300 / \mathrm{mm} .{ }^{3}\right)$ and was independent of the nature of the medium. The total yield for each medium in flask cultures was greater than in tube cultures.

Table, 4. Growth of Acanthamoeba sp. in final defined medium

Data from which log. phase of growth curve (Fig. 2) was constructed.

Amoebae/mm. ${ }^{3}$

\begin{tabular}{crrr}
\hline Days & Range (8) & Mean & s.E. \\
2 & $8-13$ & $11 \cdot 4$ & $0 \cdot 6$ \\
4 & $15-22$ & $18 \cdot 5$ & $0 \cdot 8$ \\
6 & $25-35$ & $30 \cdot 0$ & $1 \cdot 0$ \\
8 & $42-64$ & $50 \cdot 9$ & $2 \cdot 5$ \\
10 & $70-109$ & $92 \cdot 0$ & $4 \cdot 8$ \\
12 & $127-207$ & $169 \cdot 0$ & $9 \cdot 1$ \\
14 & $250-322$ & $288 \cdot 2$ & $8 \cdot 8$
\end{tabular}

Table 5. Effect of acetate and glucose on groroth in tubes

\begin{tabular}{lrrr}
\multicolumn{4}{c}{ Amoebae/mm. } \\
B.D.M.* plus & Range (5) & Mean & S.E. \\
acetate $\%(w / v)$ & & \\
0 & $13-20$ & 18 & $1 \cdot 3$ \\
0.05 & $127-181$ & 158 & $8 \cdot 5$ \\
$0 \cdot 1$ & $158-192$ & 176 & $6 \cdot 4$ \\
0.25 & $176-228$ & 193 & $9 \cdot 1$ \\
0.5 & $97-112$ & 104 & $3 \cdot 2$ \\
glucose $\%(w / v)$ & & \\
0 & & & \\
$0 \cdot 1$ & $58-87$ & 77 & $5 \cdot 2$ \\
$0 \cdot 25$ & $85-94$ & 91 & $1 \cdot 8$ \\
0.5 & $98-131$ & 120 & $7 \cdot 0$ \\
$1 \cdot 0$ & $167-200$ & 184 & $6 \cdot 1$ \\
& $226-265$ & 243 & $10 \cdot 1$
\end{tabular}

* B.D.M. amino acids as in Table $1 \times 3$, salts as in Table $1 \times 4$, trace metals as in Table 1 with citric acid replacing EDTA $5 \mathrm{ml} \%, \mathbf{B}_{12} 0.1 \mu \mathrm{g} . \%$, thiamin $0.1 \mathrm{mg} . \%$, citric acid 0.1 g. \%:

Energy compounds. The information gained in the preliminary experiments suggested that glucose $(0.25 \%, w / v)$ did not replace the effect on growth of Y.A. The question of the relative importance of acetate and glucose as energy compounds was therefore further studied. In two separate experiments amoebae were grown for 3 weeks in tubes containing defined medium to which sodium acetate or glucose was added in various concentrations. The results are shown in Table 5. The effect of acetate was approximately the same over the range of concentrations $0.05-0.25 \%(w / v)$. The threshold concentration was not determined but must be less than $0.05 \%$. Growth was significantly $(P<0.05)$ diminished at the highest concentration $(0.5 \%)$. The effect of 
glucose varied directly with the concentration. The threshold concentration under the conditions of the experiment appeared to be $0.25 \%(w / v)$. In this same experiment were included tubes containing $0 \cdot 1 \%(\mathrm{w} / \mathrm{v})$ acetate; growth (mean, 231 amoebae $/ \mathrm{mm}^{3}{ }^{3}$ ) did not differ significantly from that in the medium containing $1.0 \%$ glucose. The differences in yield of amoebae in tubes without carbohydrate in the two experiments was due to the use of an aged tracemetal solution in the first experiment, where the effect of acetate concentration was tested.

An attempt was made to test the effect of acetate and glucose on the rate of growth as distinct from the effect on yield. Media were therefore distributed in flasks and counts made at 2- or 3-day intervals up to 14 days when

Table 6. Effect of glucose and acetate on the rate of grozoth in defined medium*

\begin{tabular}{|c|c|c|c|}
\hline & \multicolumn{2}{|c|}{ Amoebae/mm. ${ }^{8} \dagger$} & \multirow{2}{*}{$\begin{array}{l}\text { Estimate of } \\
\text { M.G.T. } \neq(\text { hr.) }\end{array}$} \\
\hline & after 2 wk. & after 4 wk. & \\
\hline Without carbohydrate & 40 & 189 & 115 \\
\hline Glucose $0 \cdot 1 \%(w / v)$ & 67 & 319 & 110 \\
\hline Glucose $\mathbf{0 . 2 5} \%$ & 79 & 410 & 91 \\
\hline Glucose $0.5 \%$ & 97 & 654 & 77 \\
\hline Glucose $1.0 \%$ & 145 & 1204 & 70 \\
\hline Acetate $0.05 \%$ & 239 & contam. & 57 \\
\hline Acetate $0.1 \%$ & 292 & 741 & 55 \\
\hline Acetate $0.25 \%$ & 246 & 786 & 53 \\
\hline
\end{tabular}

* Amino acids as in Table $1, \times 3$; salts as in Table $1, \times 4$; trace metals as in Table 1 with citric acid replacing EDTA $5 \mathrm{ml} \%, \mathrm{~B}_{12} 0.1 \mu \mathrm{g} . \%$, thiamine $0.1 \mathrm{mg} . \%$, citric acid 0.1 g. \%.

$\dagger$ Mean of counts from three flasks.

$\ddagger$ M.G.T., mean generation time.

growth was expected to be logarithmic. Counts were also made at the end of 4 weeks to obtain the population size. The results of this experiment are shown in Table 6. The rate of growth was independent of the acetate concentration in the range tested, but with glucose it appeared to vary directly with the concentration. The highest concentration of glucose tested produced the largest population but the rate of growth, as indicated by the mean generation time (M.G.T.), was less than in the media containing acetate.

The $\mathrm{pH}$ value of the media at the end of 4 weeks' growth was measured electrometrically; in the flasks containing acetate it was $7 \cdot 7$, and in those containing glucose, 6.8. In a single experiment four flasks were set up with medium containing $0 \cdot 1 \%(\mathrm{w} / \mathrm{v})$ acetate. After 2 weeks' growth the $\mathrm{pH}$ of two of the flasks was decreased from 7 to 6 by the addition of $\mathrm{N}-\mathrm{HCl}$. At the end of 4 weeks' growth, the counts for the treated flasks were 1184 and 1274 amoebae $/ \mathrm{mm} .{ }^{3}$ and for the untreated, 646 and 601 amoebae/mm. ${ }^{3}$. This result showed that as the amoebae used up acetate, the accumulation of base limited the size of the population. Further, when the $\mathrm{pH}$ of the medium containing acetate was controlled, the yield with $0 \cdot 1 \%(\mathrm{w} / \mathrm{v})$ acetate was similar to that obtained with $1.0 \%$ glucose. 


\section{DISCUSSION}

The most interesting result of this work on the nutritional requirements of Acanthamoeba sp. is that only two vitamins, namely $\mathrm{B}_{12}$ and thiamine, are essential for growth. A requirement for an exogenous source of $B_{12}$ and thiamine is common among phyoflagellates and is regarded as characterizing auxotrophic algae (Provasoli, McLaughlin \& Droop, 1957). Further, when Ancanthamoeba sp. is compared with the other protozoa which can be grown in chemically defined media, namely Tetrahymena pyriformis (Kidder \& Dewey, 1951), Herpetomonas (Strigomonas) culicidarum (=Crithidia fasciculata) (Cowperthwaite, Weber, Packer \& Hutner, 1953), Leishmania tarentolae (Trager, 1957) and Strigomonas oncopelti (Newton, 1957), although all need thiamine and other vitamins, Acanthamoeba is the only one which requires $B_{12}$. A similar comparison of purine and pyrimidine requirements shows that Acanthamoeba and $S$. oncopelti are the only species which do not need an exogenous supply of purine or pyrimidine in common with auxotrophic algae. Systematic studies have shown that all the major groups of the Phytoflagellata have some representatives which are referred to as 'amoeboid' or may have an amoeboid phase in the life cycle, whereas in the Sarcodina there are amoebae such as Naegleria, which may have a flagellate phase in the life cycle. Thus morphological observations indicate that some of the Sarcodina may be derived from the Phytoflagellata, but this is the first time that physiological evidence has been presented to substantiate this view.

Although Acanthamoeba is plant-like in respect of vitamins and nucleic acid bases, it appears to be more animal-like in respect of amino acid requirements. However, until the nitrogen requirements have been fully analysed, this point must remain uncertain.

It is common experience that one of the obstacles to obtaining growth of protozoa or algae in chemically defined media lies in finding suitable concentrations of metals (Provasoli et al. 1957). In the present study Acanthamoeba sp. grew equally well in defined media containing citric acid in the range 0.05$0.2 \%(w / v)$. At the lower concentration the ratio of chelator to added metal ion (by equivalents and excluding $\mathrm{Na}^{+}$and $\mathrm{K}^{+}$) was $0 \cdot 8: 1$. When EDTA was substituted for citric acid in the same medium at a minimal concentration $(0.015 \%, w / v)$ the chelator/metal ratio was $0 \cdot 12: 1$. The poor growth obtained in this medium did not appear to be due to under-chelation, since growth was completely inhibited when the concentration of EDTA was raised to $0.03 \%$. However, since EDTA has a high affinity for $\mathrm{Ca}^{++}$and $\mathrm{Mg}^{++}$(Martell \& Calvin, 1952); the EDTA available for chelation may have been largely combined with these cations and their concentration thus decreased below the optimum for growth, at the same time leaving the concentration of the heavy metals too high. Since citric acid forms less stable chelate compounds, even when it is present in excess the concentration of metal ions will be relatively high, and a metal balance favourable to growth is more readily obtained. Citric acid was shown not to be an essential metabolite but was required to keep metals in solution. 
Neff et al. (1958) have shown that Acanthamoeba sp. is aeroboic with a relatively high rate of endogenous respiration $\left(120 \mu \mathrm{l} . \mathrm{O}_{2} / \mathrm{hr} . / \mathrm{mg} . \mathrm{N}\right.$ at $\left.28^{\circ}\right)$, and that it grows better in shallow than deep stationary cultures. It is thus probable that the slow rate of growth and low yield of amoebae obtained in screwcapped test tubes were due to $\mathrm{O}_{2}$ deficiency. The same volume of medium placed in flasks supported three to six times the population, and logarithmic growth persisted for a longer period. The observation that exponential growth stopped at about the same concentration when the amoebae were grown in different media is perhaps not surprising: the amoebae are more dense than the medium and require a surface for growth in stationary cultures. An approximate estimation shows that at this concentration the amoebae, when expanded, would cover one-half of the surface available. The rate of growth could no doubt be improved if the amoebae were grown at $28^{\circ}-30^{\circ}$ which Neff et al. (1958) have shown to be the optimum temperature.

Neff (1957) showed that Acanthamoeba sp. grew only poorly in a medium of peptone and salts unless 1.0\% (w/v) glucose was added; Reich (1935) made a similar observation with Mayorella palestinensis. Although glucose appeared to be essential for growth, neither Neff et al. (1958) nor Reich (1948) was able to show stimulation of the respiration when glucose was added to suspensions of amoebae in a Warburg apparatus. A comparison of the effect of glucose and acetate on the growth of Acanthamoeba in defined medium has shown that acetate stimulates growth, and that the rate of growth appears to be independent of the acetate concentration. Glucose also stimulates growth but the rate of growth increases with the glucose concentration; and indeed, exceptionally high concentrations of glucose are required before the rate of growth approaches that obtained with acetate. The observations on the effect of acetate on growth are consistent with the view that the undissociated acetate molecule enters amoebae readily. The observations on the effect of glucose on growth suggest that this molecule does not enter the cell through the surface but only becomes available for metabolism when ingested in food vacuoles. This suggestion is supported by the experiments of Chapman-Andresen \& Holter (1955) on glucose intake in Chaos chaos. These authors showed that uniformly ${ }^{14} \mathrm{C}$ labelled glucose entered the cell rapidly and was metabolized only when the amoebae were induced to form pinocytosis vacuoles. The fact that many algal flagellates are able to use only a limited range of nutrients for growth may be related to the impermeability of the cell membrane. With the loss of chlorophyll and a shift towards an animal mode of nutrition, either the cell membrane must become permeable to a wider range of nutrients, or the organism could become phagotrophic. Acanthamoeba is undoubtedly phagotrophic and can be seen forming food vacuoles in fluid media. Hence the stimulant effect on growth of increases in the concentration of nutrients in the medium could be explained by ingestion of the medium rather than by the diffusion or active transport of nutrients into the cell.

I wish to thank warmly Dr S. H. Hutner for hospitality at the Haskins Laboratories, New York, and for his enthusiastic instruction in methods for studying the nutrition of micro-organisms. I should also like to thank Dr M. R. Droop of the 


\section{Nutrition of Acanthamoeba}

Marine Station, Millport, for helpful discussion on chelating agents. I am also indebted to the Moray Endowment Fund of the University of Edinburgh for a grant for the purchase of apparatus and chemicals.

\section{REFERENCES}

Chapman-Andresen, C. \& Holter, H. (1955). Studies on the ingestion of ${ }^{14} \mathrm{C}$ glucose by pinocytosis in the amoeba Chaos chaos. Exp. Cell Res. (Suppl.), 3, 52.

Cowperthwaite, J., Weber, M. M., Packer, L.:\& Hutner, S. H. (1953). Nutrition of Herpetomonas (Strigomonas) culicidarum. Ann. N.Y. Acad. Sci. 56, 972.

Fisher, R. A. (1941). Statistical Methods for Research Workers, 8th ed. Edinburgh: Oliver and Boyd.

Hutner, S. H., BACH, M. K. \& Ross, G. I. M. (1956). A sugar-containing basal medium for vitamin $\mathrm{B}_{12}$-assay with Euglena; application to body fluids. J. Protozool. 3, 101.

Hutner, S. H., Provasoli, L. \& Filfus, J. (1953). Nutrition of some phagotrophic, fresh-water Chrysomonads. Ann. N.Y.Acad. Sci. 56, 852.

Kidder, G. W. \& Dewey, V. C. (1951). In Biochemistry and Physiology of Protozoa (ed. A. Lwoff), 1, 323. New York: Academic Press Inc.

Martell, A. E. \& Calvin, M. (1952). Chemistry of Metal Chelate Compounds. New York: Prentice-Hall Inc.

NEFF, R. J. (1957). Purification, axenic cultivation and description of a soil amoeba, Acanthamoeba sp. J. Protozool. 4, 176.

NefF, R. J., NefF, R. H. \& TAYLOR, R. E. (1958). The nutrition and metabolism of a soil amoeba, Acanthamoeba sp. Physiol. Zoöl. 31, 73.

Newton, B. A. (1957). Nutritional requirements and biosynthetic capabilities of the parasitic flagellate Strigomonas concopelti. J. gen. Microbiol. 17, 708.

Provasoli, L., Mclaughin, J. J. A. \& Droop, M. R. (1957). The development of artificial media for marine algae. Arch. Mikrobiol. 25, 392.

REICH, K. (1935). The cultivation of a sterile amoeba on media without solid food. J. exp. Zool. 69, 497.

ReIch, K. (1948). Studies on the respiration of an amoeba, Mayorella palestinensis. Physiol. Zoöl. 21, 390.

Trager, W. (1957). Nutrition of a haemoflagellate (Leishmania tarentolae) having an interchangeable requirement for choline or pyridoxal. J. Protozool. 4, 269. 